\title{
Deletion of the rat trpc4 gene and its influence on motivated responding for natural reward
}

\author{
William D. Klipec ${ }^{1 \star}$, Bridget Deeney ${ }^{1}$, Claire Williamson ${ }^{1}$, Kami Wenzel ${ }^{1}$, Phuong Nguyen $^{1}$, Eric \\ Ostertag $^{2} \&$ Donald C. Cooper ${ }^{3}$
}

TRPC4 ion channels are expressed extensively in corticolimbic brain regions and a subpopulation of midbrain dopamine neurons. TRPC4 knockout (KO) rats show reduced sociability and social exploration, but show no differences in simple and complex strategic learning compared to normal wild type (WT) rats. Using water reward, we found no differences between TRPC4-KO and WT rats in the break point on a progressive ratio schedule of reinforcement. Although deletion of the trpc4 gene alters social interaction/anxiety it does not appear to affect motivation for natural rewards. Current experiments are underway testing the role of trpc4 gene deletion on cocaine reward (see www.neuro-cloud,net/nature-precedings/klipec3 for updates and collaborations).

The TRPC4 channel is a nonselective cation channel modulating cellular excitability that is widely expressed in corticolimbic mammalian brain regions. These areas receive extensive input from ventral tegmantal area (VTA) dopamine (DA) neurons. ${ }^{1}$ These DA neurons modulate reward systems and stress response. We have reported that TRPC4 mRNA and protein are expressed selectively in a subpopulation of VTA DA neurons ${ }^{2}$. We reported reduced social interaction in TRPC4 KO compared to WT rats ${ }^{3}$, but we have found no differences between TRPC4 KO and WT rats in learning or performance on simple maze learning or complex strategic learning ${ }^{4}$. Here we examined the effects of deleting the trpc4 gene on motivation for water reward by comparing TRPC4 KO and WT on a progressive ratio schedule of reinforcement.

\section{RESULTS}

TRPC4 KO and WT rats were trained to lever press for water reward and then run through a progressive ratio schedule of reinforcement where the number of responses required for the next reinforcer was systematically increased after each reinforcement until the rat stopped responding for 3-minutes. The fixed ratio for the last obtained reinforcer (FRMax) was used as the break point. Fig. 1 shows that although there was a significant decline in the break point across the ten training days there were no significant differences between TRPC4 KO and WT rats.

\section{DISCUSSION}

The lack of any affect of deleting the trpc4 gene on motivation for water reinforcement or simple and complex learning tasks is surprising given the importance of DA neurons in modulating reward and the expression of TRPC4 in VTA DA neurons. These experiments used water reward, but it will be important to conduct experiments with other rewards, such as food or drugs that powerfully activate the DA system like cocaine, given the selective expression of TRPC4 channels on DA neurons.

\section{Methods/Progress and Collaborations}

The procedures for generating and genotyping the Fischer 344 TRPC4 KO and WT rats and genotyping procedures have been previously reported ${ }^{3}$.Experiments were conducted in fully automated Coulbourn operant chamber systems. Breakpoints for each rat were analyzed with a 2 x 10 split plot factorial ANOVA. All experiments were approved by the Institutional Animal Care and Use Committee at Drake University. To see detailed methods and up to date progress on this project or if you are interested in collaborating on this project visit: www.neuro-cloud.net/natureprecedings/klipec3

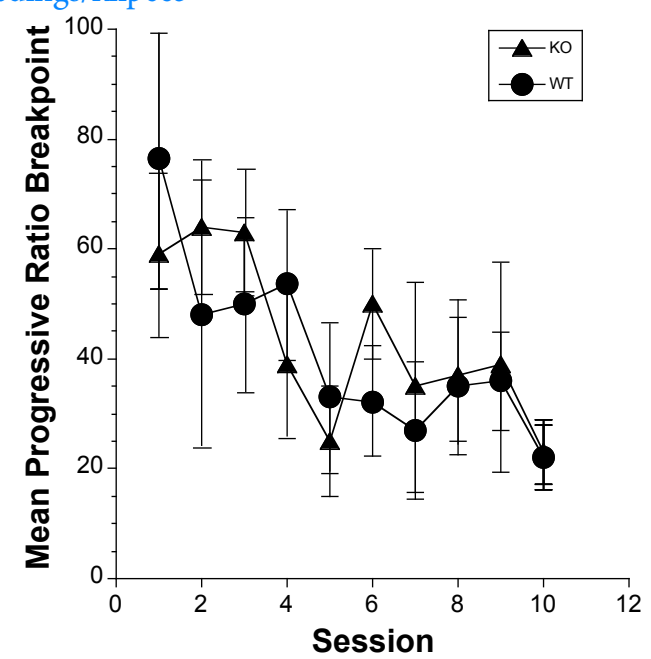

Fig. 1: Mean progressive ratio break points for TRPC4 KO and WT rats using water reinforcement. The figure shows a significant decrease in the fixed ratio breakpoint across the sessions $(\mathrm{P}<0.001)$ with no significant differences between TRPC4 KO and WT rats ( $\mathrm{P}>0.21)$. The error bars represent the $95 \%$ confidence intervals for the groups.

\section{AUTHOR CONTRIBUTIONS}

W.D.K. designed the experiments and analyzed the data. B.D., C.W., K.W. and P.N. conducted the experiments. W.D.K. and D.C.C. wrote the paper.

1. Fowler MA, et al. PLoS ONE 2(6): e573. doi:10.1371/journal.pone.0000573

2. Rasmus K, et al, Nat. Pre. <http://dx.doi.org/10.1038/ npre.2011.6367.1>

3. Illig K, et al, Nat. Pre. <http://dx.doi.org/10.1038/ npre.2011.

4. Klipec WD, et al, Nat. Pre. <http://dx.doi.org/10.1038/ npre.2012.

\footnotetext{
${ }^{1}$ Department of Psychology, Drake University, Des Moines, IA 50311, USA

2.Transposagen Biopharmaceuticals, Inc Lexington, KY 40506

${ }^{3}$ Department of Psychology and Neuroscience, Institute for Behavioral Genetics, University of Colorado, Boulder, CO 80303 USA
}

*Correspondence should be sent to william.klipec@drake.edu 